\title{
Study Title: Analysis of Biofilm Formation and Drug Resistance Profile From Sclero-Corneal wounds of The Patients Undergoing Cataract Surgery
}

\author{
Dr. Lalitha K Raju ${ }^{1}$, Dr. Shalini ${ }^{2}$, Dr. Leela $\mathrm{R}^{3}$ \\ ${ }^{1,2}$ (Department of Ophthalmology, Sankara Eye Hospital, Bangalore) \\ 3(Department of Microbiology, Sankara Eye Hospital, Bangalore)
}

\begin{abstract}
The study was aimed to assess the micro flora of sclero-corneal wounds at the end of cataract surgery before application of intra-cameral antibiotic and determine the biofilm forming ability and antimicrobial susceptibility of the isolates obtained. Thisprospective clinical study included 50 patients undergoing manual small incision cataract surgery without any complications, over a period of 6 months. In sterile environment, taking aseptic precautions wipe samples were taken from the surface of bulbar conjunctiva, corneal and scleral corneal incision following uneventful surgery. All samples were plated on various media. Positive growth was obtained from culture of 22 patients and total organisms isolated were 28. Staphylococcus aureus was the most common organism (28.6\%) followed by Coagulase negative staphylococcus (14.3\%) and Klebsiella pneumoniae (14.3\%). 15 of the isolates were weakly, 5 were moderate and 3 were strongly positive biofilm formers. $35.7 \%$ of the isolates were pan-drug resistant. There was a significant correlation between diabetic and positive culture growths $(p=0.0118)$. Multiple factors can lead to post- surgical endophthalmitis with considerable local variation in the causative organisms and the antibiotic susceptibility. All these aspects should be kept in mind to avoid the infection.
\end{abstract}

Keywords: antibiotic resistance, biofilm,cataract,endophthalmitis.

\section{Introduction}

Post-operative endophthalmitis is defined as severe inflammation involving both the anterior and posterior segments of the eye following intraocular surgery [1]. It is one of the most serious, sight-threatening complication of cataract surgery that every ophthalmic surgeon and patient strives to avoid. Its overall incidence varies according to the technique and region of the world, ranging from $0.028 \%$ to $0.2 \%[2,3,4]$. Though the overall incidence is low, given the considerable number of cataract surgeries performed each year, a substantial number of patients are affected by this devastating complication.

The exact pathophysiological mechanisms causing endophthalmitis is not completely understood, in most cases, it is due to microbial contamination either from patient's microbiota or from contaminated surgical equipment and surrounding operating environment [5].Based on numerous studies, the primary source is considered to be bacteria from the patient's ocular surface (cornea, conjunctiva) or adnexa (lacrimal glands, eyelids, and extraocular muscles)[6,7] reflecting the fact, that aqueous humorcontamination with surface bacterial flora is the main event in pathogenesis[8] which varies between 5-43\% [9]. Further, one of the most important factors contributing to the virulence of bacteria is biofilm-formation capacity [10] on the surface of IOLs or posterior capsule which prevent the clearance and provide a higher resistance to antimicrobial therapy.

The micro-organisms most commonly recognized in post-operative endophthalmitis may vary considerably with regions of the world and so is their susceptibility to the antimicrobial agents.Thus, the objective of our study was to assess the bacterial flora of the sclera-corneal wounds at the end of uneventful cataract surgery before intra-cameral antibiotic use, to test their potential for biofilm formation and their antibiotic resistance. At a time, where spread of antibiotic resistance is high, understanding this may help in an effective antibiotic strategy and would be critical to improving patient outcome.

\section{Material and Methods}

\subsection{Sampling}

This is a prospective clinical study. The descriptive study included 50 patients. It was carried out at the Community outreach section of Sankara Eye Hospital, Bangalore where people were screened and brought. All the patients included in the study underwent uneventful cataract extraction by manual small incision cataract surgery. The inclusion criteria included patients with age more than 18 years diagnosed with cataract without 
Study Title: Analysis Of Biofilm Formation And Drug Resistance Profile From ...

any other eye disease and patients without any complications during the surgery. Patients with complicated surgery and patients using topical or systemic antibiotics prior to surgery were excluded. Informed consent was taken from all patients and the study was approved by the local Ethical Committee. Detailed routine preoperative assessment was done for each patient.

Patients underwent cataract surgery in sterile operating theatres that had air filters. Peri-bulbar anesthesia was given in the selected eye and Povidone eye drop (5\%) instilled. Peri-orbital,hemi-facial skin, eyelids and eyelashes were thoroughly disinfected with $10 \%$ Povidone-Iodine. The surgical surface was covered with single use, sterile ophthalmic drape and the eye speculum was placed cautiously by the operating surgeon to keep the eyelids and eyelashes under the drape. After an uncomplicated cataract extraction before administration of intra-cameral antibiotics, taking aseptic precautions wipe samples were taken from the bulbar conjunctival surface, corneal surface and scleral- corneal wound with sterile transport swabs. Samples were immediately transferred to the Department of Microbiology for further analysis.

Samples were immediately stored at 4 degrees centigrade and were cultured within 24 hours. All samples were plated on nutrient agar, blood agar, chocolate agar, MacConkey agar and Sabouraud- dextrose agar. Plates were incubated at $37{ }^{\circ} \mathrm{C}$ for 24 to 48 hours. Significant colony growth on all these media for all the three samples was analyzed and identified by standard microbiological and biochemical tests such as colony morphology, Gram's stain, catalase test, oxidase test, slide/ tube coagulase test, fermentation of mannitol.
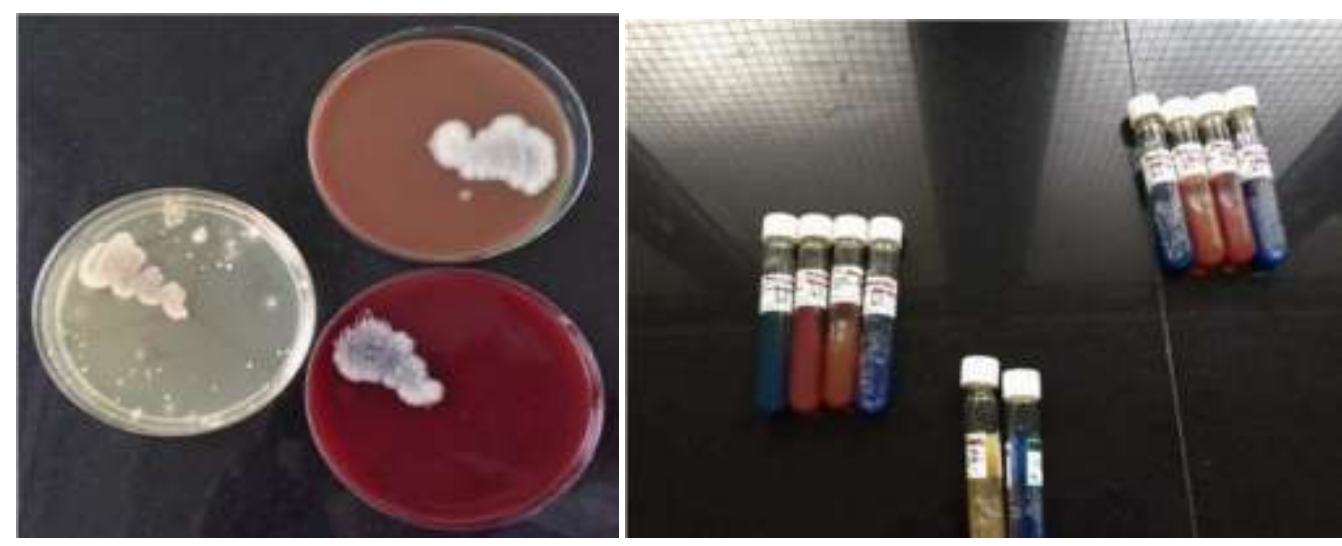

Figure 1: Culture plates showing positive growthFigure 2: Various biochemical tests

\subsection{Characterization of biofilms}

There are various techniques to evaluate biofilm such as Tissue culture plate method[11] tube method (TM) [12,13] Congo red agar (CRA) [14] bioluminescent assay[15] and light or fluorescence microscopic examination [16]. Weemployed the simple technique of Tube method. Here, $10 \mathrm{ml}$ of BHIB (brain heart infusion broth) with $1 \%$ glucose was inoculated with a loopful of organism to be tested from overnight culture on nutrient agar individually. Broths were incubated at 37 degrees centigrade for 24 hours. The culture tubes were decanted and washed with phosphate buffered saline at $\mathrm{pH}$ 7.3. The tubes were dried and stained with $0.1 \%$ crystal violet. Excess stain was washed with deionized water. Tubes were dried in inverted position until dry and then results interpreted.

In positive biofilm, a visible stained film was seen lining the wall and bottom of the tube. Ring formation at the liquid interface was not indicative of biofilm formation. Tubes were examined and the biofilm formation was scored as 0- absent, 1- weak, 2- moderate and 3- strong.

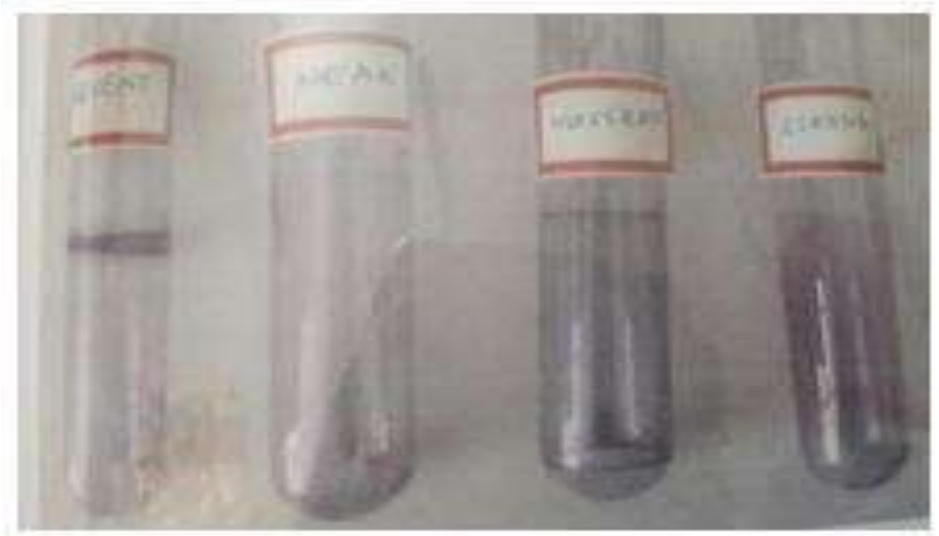

Figure 3: Biofilm formation 
Study Title: Analysis Of Biofilm Formation And Drug Resistance Profile From ...

\subsection{Antimicrobial susceptibilities}

This was done for each isolate by Kirby Bauer disc diffusion method. The surface of Mueller Hinton agar plate was inoculated with $\log$ phase cultures with the turbidity corresponding to $0.5 \mathrm{McF}$ arland turbidity standard. Antibiotics discs (Hi Media) were placed on the plate, and incubated at $37{ }^{\circ} \mathrm{C}$.After 18-24 hours, the diameter of clear zone around the disc was measured under transmitted light with measuring scale and results interpreted as susceptible or resistant as per CLSI guidelines [17]. The antibiotics tested include amikacin (30 $\mathrm{mcg}$ ), amoxyclav $(30 \mathrm{mcg})$, ampicillin $(10 \mathrm{mcg})$, ceftazidime $(30 \mathrm{mcg})$, ceftizoxime $(30 \mathrm{mcg})$, ceftriaxone $(30 \mathrm{mcg})$, cefotaxime $(30 \mathrm{mcg})$, cefuroxime $(30 \mathrm{mcg})$, co-trimoxazole $(25 \mathrm{mcg})$, ciprofloxacin $(5 \mathrm{mcg})$, gatifloxacin (5mcg), gentamicin (10 mcg), imipenem (10 mcg), levofloxacin $(5 \mathrm{mcg})$, linezolid (15mcg), moxifloxacin $(5 \mathrm{mcg})$, ofloxacin $(5 \mathrm{mcg})$, oxacillin $(1 \mathrm{mcg})$, penicillin (10 units) and tobramycin $(10 \mathrm{mcg})$.

Standard American Type Culture Collection (ATCC) bacteria Staphylococcus aureus ATCC 25923, Pseudomonas aeruginosa ATCC 27853 and E. Coli ATCC 25922 were used for quality control

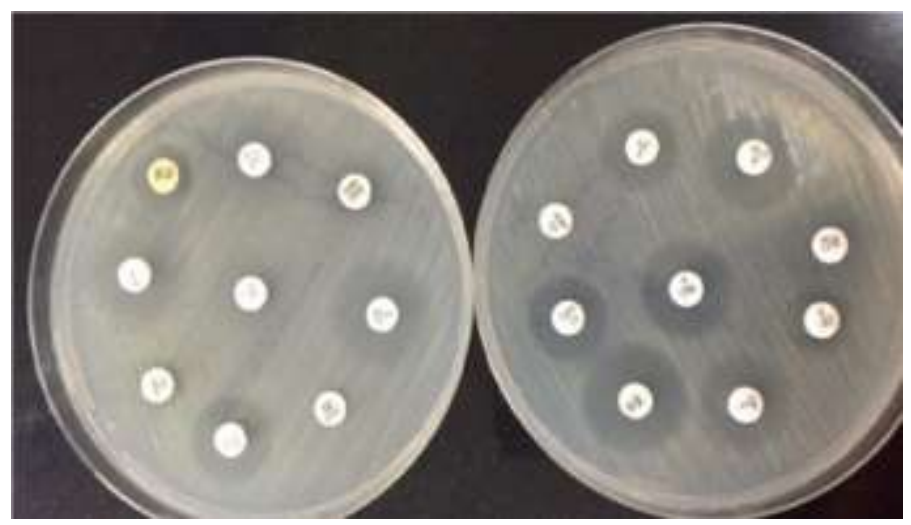

Figure 4: Antiobiotic sensitivity test

\section{Statistical Analysis}

Data analysis was done with the help of computer using Statistical Package for Social Sciences (SPSS Inc., Chicago, IL, version 22 for Windows).Using this software range, frequencies, percentages, means, standard deviations, chi square, correlation coefficient and 'p' values were calculated. Chi-square test and Pearson's correlation were performed between biofilm formation and antibiotic resistance. Differences were considered statistically significant if $\mathrm{p}$ value $<0.05$

\section{Results}

Our study included 50 patients who underwent uneventful cataract surgery. There were 25 males and 25 female patients with mean age of 61.9 years (the age ranged from 41-80 years).

Positive growth was observed in cultures of 22 eyes (44\%). Few samples showed multiple bacterial species. We obtained 28 isolates from the positive cultures. Of the 50 cases, 9 patients were diabetic and 2 were both diabetic and hypertensive. Other systemic conditions associated included hypertension (9 patients), chronic obstructive pulmonary disease (4 patients) and heart disease ( 2 patients) whereas 24 patients had no other identified systemic associations. It was observed that there was statistically significant correlation between culture positive cases obtained from diabetic and non-diabetic cases $(\mathrm{p}=0.0118)$.

\begin{tabular}{|c|c|c|c|c|}
\hline \multirow{3}{*}{ Diabetes Mellitus } & \multicolumn{4}{|c|}{ Culture } \\
\hline & \multicolumn{2}{|c|}{$\begin{array}{l}\text { Positive } \\
\text { cases }\end{array}$} & \multicolumn{2}{|c|}{ Negative cases } \\
\hline & No. & $\%$ & No. & $\%$ \\
\hline Present(11) & 9 & 81.8 & 2 & 18.2 \\
\hline Absent (39) & 13 & 33.3 & 26 & 66.7 \\
\hline $\begin{array}{l}\text { Chi square } \\
\text { 'p' }\end{array}$ & \multicolumn{4}{|c|}{$\begin{array}{l}6.3363 \\
\text { 0.0118 Significant }\end{array}$} \\
\hline
\end{tabular}

Table 1: Growth in Diabetic and non- diabetic case

Based on the various biochemical and microbiological tests performed, the various isolates obtained were identified as- Staphylococcus aureus (28.6\%), Coagulase negative staphylococcus (14.3\%), Klebsiella pneumoniae (14.3\%), Pseudomonas aeruginosa (14.3\%), Bacillus cereus (7.1\%), Aspergillus niger (3.6\%), Acinetobacter baumanii (3.6\%), Escherichia coli (3.6\%), Moraxella catarrhalis (3.6\%). All the isolates were individually screened on their ability to form biofilm, based on the tube test method. A total of 23 out of 28 
Study Title: Analysis Of Biofilm Formation And Drug Resistance Profile From ...

isolates $(82.14 \%)$ were biofilm formers that ranged from weak to strong. Majority of the isolates were weak biofilm formers $(53.5 \%), 17.9 \%$ of the isolates were moderate whereas only $10.7 \%$ of the isolates were strong biofilm formers.
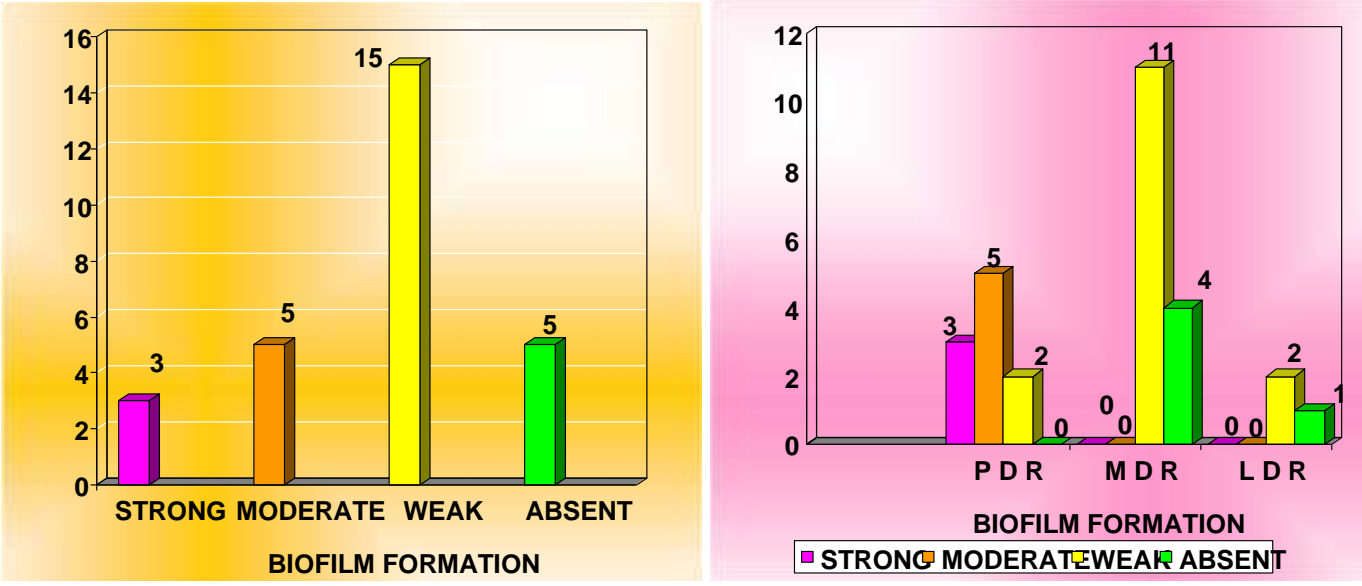

Graph 1: Biofilm assay Graph 2: Correlation between biofilm formers and drugresistance Further,the antimicrobial susceptibility tests were performed for all the isolates individually with the wide range of antibiotics as described above. The highest resistance was observed for Ampicillin that was seen in $96.4 \%$ of the isolates. Next in order, around $71.4 \%$ isolates were resistant to Ceftriaxone, $67.9 \%$ to Amoxycillin /Clavulanic acid, $46.4 \%$ to Co-Trimoxazole, $31.8 \%$ to Cefuroxime, $28.5 \%$ were found resistant to the aminoglycoside Tobramycin, 25\% to both Oxacillin and Penicillin, and $7.1 \%$ to Amikacin. All the isolates were susceptible to Imipenem. $4.5 \%$ isolates were resistant to Gentamicin.Among the different generations offluoroquinolones used, $22.7 \%$ of the isolates were Moxifloxacin resistant, $18.18 \%$ resistant to Gatifloxacin, $13.6 \%$ to Levofloxacin whereas $9.09 \%$ to Ofloxacin and Ciprofloxacin.Isolates showing resistance to five or more antibiotic classes were characterized as pan drug resistant (PDR) and those showing resistance to three or more drugs were characterized as multiple drug resistant organisms (MDR) [18]. Around 35.7\% of the total isolates were Pandrug resistant and $53.6 \%$ were Multi-drug resistant. Chi- square analysis revealed significant co-relation between Pan drug resistant and biofilm formers $(\mathrm{p}<0.0001$, correlation coefficient $=0.7072)$.

\begin{tabular}{|c|c|c|c|c|c|c|c|c|c|}
\hline \multirow{3}{*}{$\begin{array}{l}\text { Antibiotic Resistance } \\
\text { type in isolates }\end{array}$} & \multirow{3}{*}{$\begin{array}{l}\text { Number } \\
\text { Of cases }\end{array}$} & \multicolumn{8}{|c|}{ Biofilm formation } \\
\hline & & \multicolumn{2}{|c|}{ Strong } & \multicolumn{2}{|c|}{ Moderate } & \multicolumn{2}{|c|}{ Weak } & \multicolumn{2}{|c|}{ Absent } \\
\hline & & No. & $\%$ & No. & $\%$ & No. & $\%$ & No. & $\%$ \\
\hline Pan Drug Resistant & 10 & 3 & 30.0 & 5 & 50.0 & 2 & 20.0 & - & - \\
\hline Multiple Drug Resistant & 15 & - & - & - & - & 11 & 73.3 & 4 & 26.7 \\
\hline Low Drug Resistant & 3 & - & & - & - & 2 & 66.7 & 1 & 23.1 \\
\hline $\begin{array}{l}\text { Chi square } \\
\text { 'p' } \\
\text { Correlation Coefficient }\end{array}$ & \multicolumn{9}{|c|}{$\begin{array}{l}16.43 \\
<0.0001 \text { (Significant) } \\
0.7072 \text { (Strong positive correlation) }\end{array}$} \\
\hline
\end{tabular}

Table 2:Correlation between Biofilm formation and antibiotic susceptibility in isolates

\section{Discussion}

It is fortunate that post-surgical endophthalmitis is rare, but due to the potentially devastating complications it can lead to, infection prophylaxis, diagnosis, and treatment have always been the subject of ongoing research and debate. Various studies reveal, most cases are due to bacteria entering the eye at the time of surgery [19],so it is believed that the chief source of infection is usually the patient's own microbial flora from the conjunctiva, eyelids and lacrimal sac [20].The most commonly implicated organisms causing endophthalmitis worldwide are Coagulase negative staphylococci (33-77\%), Staphylococcus aureus (10-21\%), Streptococci (9-19\%), Gram negative bacteria including Pseudomonas aeruginosa (6-22\%) and fungi (up to 8\%) [21]. An evidence-based review of the literature concluded that preoperative povidone-iodine prophylaxis was the single most effective means for reducing the risk for postoperative endophthalmitis (decreasing microbial load up to $91 \%$ )[22,23].Still a considerable number of positive culture have been obtained from the post-surgery patients in spite of the use of povidone-iodine in many studies, to name a few such as those done by Quiroga et al[24] and Kumar et al[25], emphasizing other methods should be sought to further reduce conjunctival flora. . 
Study Title: Analysis Of Biofilm Formation And Drug Resistance Profile From ...

In this study, the most common organism isolated was Staphylococcus aureus (28.6\%)followed by Coagulase negative Staphylococcus (14.3\%) and Pseudomonas (14.3\%). The current findings are in agreement with earlier reports that wide microbial spectrum of endophthalmitis is highly region specific [18, 19].We obtained higher percentage of Klebsiella pneumoniae in our study (14.3\%), this is in accordance to the studies done by Lundstorm[26] et al and Jumbulingam[5] et al in Southeast Asia and India, where rates of gram negative infections have been seen to be as high as $53 \%$ and of fungal infections up to $16 \%$.

We found,a significantly higher rate of positive culture in diabetic than non- diabetic patients. This is in accordance to studies done by Kivanc et al [27] Fernandez R et al [28] Biofilm forming ability of the isolateswas studied. Out of 28 isolates, 23 were biofilm formers, though majority were weak biofilm formers (15 out of 23).A biofilm is defined as a functional consortium of microorganisms organized within an extensive exopolymer matrix [29]. Biofilms can form on both abiotic (such as intraocular lenses, posterior contact lenses, scleral buckles, conjunctival plugs, lacrimal intubation devices and orbital implants) or biotic surfaces (such as posterior capsule and corneal stroma) and are associated with a large share of human infections.It is also noteworthy that the bacteria in biofilms are more resistant to antiseptics, antibiotics, and host defenses [30].Physiological heterogeneity, complicated structure, elevated expression of efflux and relative anaerobiasis at the deeper layers of biofilm may be responsible for this high resistance.Cataract surgery with intra-ocular lens provides platform for the development of infections involving biofilm that are refractory to therapy.

The other aspect of this study is,antibiotic profile of the organisms. The strong biofilm formers were pan-drug resistant. This is in accordance with the study done by Kivanc et al[27] and Katiyar et al [18]. We also noted all the isolates were susceptible to Imipenem. Highest drug resistance was for Ampicillin and Ceftriaxone. The two noteworthy observations were- high resistance to second generation cephalosporin Cefuroxime (31.8\%), which was recommendedas the intra-cameral antibiotic prophylaxis for post-operativeendophthalmitis by European Society of Cataract and Refractive Surgeons (ESCRS) [31]. Secondly, resistance to fourth generation Moxifloxacin and Gatifloxacin was found to be more than second generation Ofloxacin and Ciprofloxacin indicating rise in multi-step topoisomerase mutants. Similar results were obtained in some other recent studies also $[18,27]$. Fluoroquinolones have wide antibacterial spectrum and are one of the most popularly used drugs in ophthalmology but recent decades have seen increase in the resistance rate especially to Moxifloxacin [32]. Another noticeable result was high susceptibility (95.5\%) to bactericidal antibiotic Gentamicin. There are several prophylactic measures employed at the end of cataract surgery such as intracameral and sub-conjunctival antibiotic injection and instillation of Povidone-iodine eye drops (5\%) before patching the eye owing to very low incidence of post-operative endophthalmitis at our institute and hence may be the reason for no positive case from the sample studied till date.

\section{Conclusion}

The present study is just a glimpse of the microbial load following cataract surgery of the local area. These micro-organisms had the ability to form biofilm and showed resistance to diverse classes of antibiotics including some of the very frequently used drugs.To conclude, in each endophthalmitis case, the true challenge lies in taking right and quick decisions in order to reinstate the best vision as possible. In the era of spread of antibiotic resistance and increasing knowledge regarding biofilm, it is of vital importance that our patients receive the greatest benefit from the antibiotics available. Further research and studies are required in developing strategies to prevent or eradicate the biofilm at the site of infection.

\section{Acknowledgement \\ Technical support of Mr. Javeed Ahmed N is acknowleged.}

\section{References}

[1]. Zagaria M. Postoperative endophthalmitis after cataract surgery. US Pharm, 41(4), 2016, 8-11.

[2]. Taban M, Behrens A, Newcomb RL, Nobe MY, Saedi G, Sweet PM, McDonnell PJ. Acute endophthalmitis following cataract surgery: A systematic review of the literature. Arch. Ophthalmol, 123, 2005, 613-620.

[3]. Wykoff CC, Parrott MB, Flynn HW Jr, Shi W, Miller D, Alfonso EC. Nosocomial acute-onset postoperative endophthalmitis at a university teaching hospital (2002-2009). Am. J. Ophthalmol, 150, 2010, 392-398.

[4]. West ES, Behrens A, McDonnell PJ, Tielsch JM., Schein OD. The incidence of endophthalmitis after cataract surgery among the U.S. Medicare population increased between 1994 and 2001. Ophthalmology, 112, 2005, 1388-1394.

[5]. Jambulingam M, Parameswaran SK, Lysa S, Selvaraj M, Madhavan HN. A study on the incidence, microbiological analysis and investigations on the source of infection of postoperative infectious endophthalmitis in a tertiary care ophthalmic hospital: An 8year study. Indian J. Ophthalmol, 58, 2010, 297-302.

[6]. Buzard K, Liapis S. Prevention of endophthalmitis. J Cataract Refract Surg, 30, 2004, 1953-1959.

[7]. Speaker MG, Milch FA, Shah MK. Role of external bacterial flora in the pathogenesis of acute postoperative endophthalmitis. Ophthalmology, 98, 1991, 639-649.

[8]. Durand ML. Endophthalmitis. ClinMicrobiolInfect, 19, 2013, 227-234

[9]. Dickey JB, Thompson KD, Jay WM. Anterior chamber aspirate cultures after uncomplicated cataract surgery. Am J Ophthalmol, 112, 1991, 278-282. 
Study Title: Analysis Of Biofilm Formation And Drug Resistance Profile From ...

[10]. Zegans ME, Becker HI, Budzik J, O’Toole G. The role of bacterial biofilms in ocular infections. DNA Cell Biol, 21(5-6), 2002, 415-420

[11]. Christensen GD, Simpson WA, Younger JA, Baddour LM, Barrett FF, Melton DM, et al. Adherence of cogulase negative Staphylococi to plastic tissue cultures:a quantitative model for the adherence of staphylococci to medical devices. J ClinMicrobiol, 22, 1985, 996-1006.

[12]. Christensen GD, Simpson WA, Bisno AL, Beachey EH. Adherence of slime-producing strains of Staphylococcus epidermidis to smooth surfaces. Infect Immun, 37, 1982, 318-326.

[13]. Mathur T, Singhal S, Khan S, Upadhyay DJ, Fatma T, Rattan A. Detection of biofilm formation among the clinical isolates of staphylococci: An evaluation of three different screening methods. Ind J Med Microbiol, 24, 2006, 25-29.

[14]. Freeman DJ, Falkiner FR, Keane CT. New method for detecting slime production by coagulase negative staphylococci. J ClinPathol, 42, 1989, 872-874

[15]. Donlan RM, Murga R, Bell M, Toscano CM, Carr JH, Novicki TJ, et al. Protocol for detection of biofilms on needleless connectors attached to central venous catheters. J ClinMicrobiol, 39, 2001, 750-753.

[16]. Zufferey J, Rime B, Francioli P, Bille J. Simple method for rapid diagnosis of Catheter-associated infection by direct Acridine orange staining of catheter tips. J ClinMicrobiol, 26, 1988, 175-177.

[17]. Clinical and Laboratory Standards Institute. Performance Standards for Antimicrobial Susceptibility Testing; seventeenth informational supplement. 19th ed., Vol. 1. Performance Standards for Antimicrobial Disk Susceptibility Tests: CLSI document M100-S17. M2-A9. Approved Standard; 2007, pp. 27

[18]. KatiyarR,Vishwakarma A, KaisthaSD.Analysis of biofilm formation and antibiotic resistance of microbial isolates from intraocular lens following conventional extracapsular cataract surgery. International Journal of Research in Pure and applied Microbiology, 2(2), 2012, 20-24

[19]. Speaker MG, Milch FA, Shah MK, Eisner W, Kreiswirth BN. Role of external flora in the pathogenesis of acute postoperative endophthalmitis. Ophthalmology, 98, 1991, 639-645

[20]. Bannerman TL, Rhoden DL, McAllister SK, Miller JM, Wilson LA. The source of coagulase-negative staphylococci in the Endophthalmitis Vitrectomy Study: A comparison of eyelid and intraocular isolates using pulsed-field gel electrophoresis. Arch Ophthalmol Chic Ill 1960, 115(3), 1997 Mar, 357-361.

[21]. Barry P, Cordovés L, Gardner S. ESCRS guidelines for prevention and treatment of endophthalmitis following cataract surgery: data, dilemmas and conclusions. EurSoc Cataract Refract Surg Dublin Irel [Internet]. 2013 [cited 2015 Nov 15].

[22]. Ciulla TA, Starr MB, Masket S. Bacterial endophthalmitis prophylaxis for cataract surgery: an evidence-based update. Ophthalmology, 109, 2002, 13-24.

[23]. Apt L, Isenberg S, Yoshimori R, Paez JH. Chemical preparation of the eye in ophthalmic surgery .III:effect of povidone-iodine on the conjunctiva. Arch Ophthalmol, 102(5), 1984, 728-729.

[24]. Quiroga LP, Lansingh V, Laspine F et al. A prospective study demonstrating the effect of $5 \%$ povidone iodine application for anterior segment intraocular surgery in Paraguay. Arq Bras Oftalmol, 73(2), 2010, 125-128.

[25]. Kumar MA, Kurien SS, Selvaraj S et al. Comparison of different techniques of cataract surgery in bacterial contamination of the anterior chamber in diabetic and non-diabetic population. Indian J Ophthalmol, 60(1), 2012, 41-44.

[26]. Lundström E, Friling PM. Risk factors for endophthalmitis after cataract surgery: predictors for causative organisms and visual outcomes. J Cataract Refract Surg, 41, 2015, 2410-2416.

[27]. Kivanc SA, Kivanc M, Bayramlar H. Microbiology of corneal wounds after cataract surgery: biofilm formation and antibiotic resistance patterns. J Wound Care, 25(1), 2016, 14-19.

[28]. Fenandez R,Rebolledo L,Martinez GM,Alarcón TM,Cortés V C. The conjunctival bacterial pattern of diabetics undergoing cataract surgery. Eye, 24(5), 2010 May, 825-834

[29]. Costerton JW, Cheng KJ, Geesey GG, Ladd TI, Nickel JC, Dasgupta M, Marrie TJ. Bacterial biofilms in nature and disease. Annu Rev Microbiol, 41, 1987, 435-464.

[30]. Askari E, Soleymani F, Arianpoor A, Tabatabai SM, Amini A, Naderinasab M. Epidemiology of mecA-Methicillin Resistant Staphylococcus aureus (MRSA) in Iran: A Systematic Review and Metaanalysis. Iran J Basic Med Sci., 15(5), 2012, 1010-1019.

[31]. ESCRS Endophthalmitis Study Group. Prophylaxis of postoperative endophthalmitis following cataract surgery: results of the ESCRS multicenter study and identification of risk factors. J Cataract Refract Surg, 33, 2007, 978-988.

[32]. Alexandrakis G, Alfonso EC, Miller D. Shifting trends in bacterial keratitis in south Florida and emerging resistance to fluroquinolones. Ophthalmology, 107(8), 2000, 1497-1502. 